\title{
Mediterranean Diet and Magnetic Resonance Imaging-Assessed Cerebrovascular Disease
}

\author{
Nikolaos Scarmeas, MD ${ }^{1,2,3}$, José A. Luchsinger, MD ${ }^{1,4}$, Yaakov Stern, PhD ${ }^{1,2,3}$, Yian Gu, \\ $\mathbf{M D}^{1,2}$, Jing He, $\mathbf{P h D}^{5}$, Charlie DeCarli, $\mathbf{M D}^{5}$, Truman Brown, $\mathbf{P h D}^{6}$, and Adam M. Brickman, \\ $\mathrm{PhD}^{1,2,3}$ \\ ${ }^{1}$ Taub Institute for Research in Alzheimer's Disease and the Aging Brain, University of California \\ at Davis, Sacramento, CA \\ ${ }^{2}$ Gertrude H. Sergievsky Center, University of California at Davis, Sacramento, CA \\ ${ }^{3}$ Department of Neurology, University of California at Davis, Sacramento, CA \\ ${ }^{4}$ Department of Medicine, University of California at Davis, Sacramento, CA \\ ${ }^{5}$ Radiology, Columbia University, New York, NY \\ ${ }^{6}$ Department of Neurology, University of California at Davis, Sacramento, CA
}

\begin{abstract}
Objective-Cerebrovascular disease is 1 of the possible mechanisms of the previously reported relationship between Mediterranean-type diet (MeDi) and Alzheimer's disease (AD). We sought to investigate the association between MeDi and MRI infarcts.
\end{abstract}

\begin{abstract}
Methods-High-resolution structural MRI was collected on 707 elderly 65 years or older community residents of New York with available dietary assessments administered an average of 5.8 years (3.22 standard deviations [SDs]) before the MRI. Participants were divided into 3 groups of adherence to $\mathrm{MeDi}$ (low, middle, and high tertiles). We examined the association of increasing adherence to MeDi with presence of infarcts on MRI. Models were run without adjustment, adjusted for basic demographic and clinical factors, and adjusted for vascular risk factors.
\end{abstract}

\begin{abstract}
Results-A total of 222 participants had at least 1 infarct. In the unadjusted model, compared to the low adherence group, those in the moderate MeDi adherence group had a 22\% reduced odds of having an infarct (odds ratio [OR], 0.78; 95\% confidence interval [CI], 0.55-1.14), while participants in the highest MeDi adherence group had a 36\% reduced odds (OR, 0.64; 95\% CI, 0.42-0.97; $p$ for trend $=0.04$ ). In adjusted models, the association between MeDi adherence and MRI infarcts remained essentially unchanged. The association of high MeDi adherence with infarcts was comparable to that of hypertension ( $40 \%$ reduced probability), did not vary by infarct size or after excluding patients with dementia $(n=46)$ or clinical strokes $(n=86)$. There was no association between $\mathrm{MeDi}$ and white matter hyperintensities.
\end{abstract}

Interpretation-Higher adherence to the $\mathrm{MeDi}$ is associated with reduced cerebrovascular disease burden.

\footnotetext{
(C) 2011 American Neurological Association

Address correspondence to Dr Scarmeas, Columbia University Medical Center, 622 West 168th Street, PH 19th floor, New York, NY 10032.ns257@columbia.edu.

Potential Conflicts of Interest

C.D. has grant(s) pending from the NIH. J.H. has received grant(s) from the NIH. J.A.L. has received grant(s) and has grant(s) pending from the NIH. N.S. has received grant(s) from the NIH and has grant(s) pending from the NIH and the Alzheimer's

Association. All other authors have nothing to report.
} 
We previously reported that higher adherence to a Mediterranean-type diet (MeDi) is associated with lower Alzheimer's disease (AD) ${ }^{1-3}$ and mild cognitive impairment ${ }^{4}$ risk. One of the possible mechanisms through which the MeDi may be exerting its protective effect for AD could be vascular: (1) higher adherence to a MeDi has been associated with lower risk for obesity, ${ }^{5-9}$ the metabolic syndrome,${ }^{10-12}$ dyslipidemia, ${ }^{13}$ hypertension, $, 5,13,14$ abnormal glucose metabolism, ${ }^{5}$ diabetes, ${ }^{15-17}$ and coronary heart disease, ${ }^{18-21}$ whereas (2) the evidence for contribution of vascular risk factors in AD risk is growing. ${ }^{22-24}$ Therefore, higher adherence to a MeDi could be related to lower cerebrovascular disease and hence lower dementia rates.

Nevertheless, in our previous investigation of this question, vascular comorbidity as ascertained by medical history did not seem to mediate the association between MeDi and $\mathrm{AD},{ }^{1}$ suggesting that nonvascular mechanisms, such as oxidation or inflammation may be playing a role. Another possible explanation could be that clinically assessed vascular variables lack sufficient sensitivity ${ }^{25,26}$ because they are proxies for cerebrovascular disease rather than direct measurements, biasing our results toward the null hypothesis. Indeed, sensitivity of self-reported stroke has been found to be low in our cohort using imaging as the gold standard. ${ }^{26}$ Therefore, it is possible that a mediating cerebrovascular effect could be detected if measurement of cerebrovascular comorbidity is performed with use of instruments or biomarkers more accurate and more biologically proximal to either vascular disease or to vascular-mediated neural damage. Magnetic resonance imaging (MRI) provides the capacity to visualize and quantify these changes in vivo.

At the same time, despite the potentially protective effects for stroke of many elements of the MeDi (fruits, vegetables, whole grains, fish, olive oil, low saturated fatty acids, moderate alcohol, etc.), ${ }^{27-33}$ the association between MeDi and stroke has received extremely limited attention in the previous literature with only a single study in women having been published. ${ }^{34}$ To our knowledge, the association between MeDi and clinical stroke has not been examined for men or for non-Caucasians, while there have been no investigations of the association between MeDi and cerebrovascular disease assessed with brain imaging.

In an attempt to shed some preliminary light on this topic, we examined the association between MeDi and MRI brain infarcts among participants of the Washington Heights/ Hamilton Heights Columbia Aging Project (WHICAP) imaging substudy, a populationbased study of elderly 65 years or older in New York. We hypothesized that higher adherence to the MeDi would be associated with lower burden of cerebrovascular disease at brain MRI.

\section{Patients and Methods}

\section{Sample}

WHICAP is a large-scale community-based project of aging and dementia based in upper Manhattan. The source cohort include was identified (via ethnicity and age stratification processes) from a probability sample of Medicare beneficiaries aged 65 years or older, residing in an area of 3 contiguous census tracts within a geographically-defined area of northern Manhattan. ${ }^{2,35-38}$ The WHICAP study has combined epidemiological, neuropsychological, neurological, and medical approaches to understand the antecedents, biological risk factors, genetics, and course of cognitive aging and dementia for over 20 years. The current cohort represents a combination of 2 cohorts from 2 recruitment waves in 1992 and 1999. Description of the recruitment and sampling strategies for the 2,776 participants that comprise the ongoing WHICAP cohort have been provided in detail elsewhere. ${ }^{39}$ 
In 2004, we began systematic collection of high-resolution neuroimaging data on ongoing WHICAP participants who did not meet criteria for dementia at their previous follow-up visit, as described in detail elsewhere (WHICAP imaging study). ${ }^{40}$ The MRI acquisition took place at a visit contemporaneous with the second follow-up assessment of the WHICAP cohort recruited in 1999. A total of 769 WHICAP participants received MRI scans. MeDi score could not be calculated (due to either missing or incomplete dietary evaluations) for $7 \%$ of those who received MRI and for 5\% of those who were eligible but refused participation in the MRI component of the parent study $(p=0.19)$. Those who received MRI scans were 1 year younger and were more likely to be men and African Americans but were otherwise similar compared with those who were eligible but refused participation in the MRI component of the parent study (for more details see Brickman and colleagues ${ }^{40}$ ).

For 62 of 769 subjects either MRI stroke assessments were not available or dietary evaluations were absent or incomplete (preventing MeDi score calculation), leaving 707 participants as the final analysis sample for this study. The 707 subjects included in the study were more likely to be African Americans (35\% vs 22\%) or Whites (27\% vs $21 \%$ ) and less likely Hispanics (36\% vs 55\%) ( $p=0.03$ ) but were otherwise demographically and clinically (age, $p=0.25$; sex, $p=0.07$; education, $p=0.09$; body mass index, $p=0.88 ; \varepsilon 4$ allele, $p=0.21$; smoking, $p=0.48$; diabetes, $p=0.38$; hypertension, $p=0.51$; heart disease, $p=0.66$ ) similar compared with those 62 subjects with missing either dietary or MRI stroke assessments.

\section{Clinical Evaluation}

At each assessment, each participant underwent an in-person interview of general health and functional ability followed by a semiaverage structured standardized assessment, including medical history, physical and neurological examination, and a neuropsychological battery that included measures of memory, orientation, language, abstract reasoning, and visuospatial ability. ${ }^{41}$ The neuropsychological test battery and its validity in the diagnosis of dementia has been described in detail in a previous publication. ${ }^{41}$ All participants received structured neurologic, medical and functional assessments. The diagnosis of dementia or its absence was based on standard research criteria ${ }^{42}$ and was established using all available information (except the MRI results) gathered from the initial and follow-up assessments and medical records at a consensus conference of physicians, neurologists, neuropsychologists, and psychiatrists.

\section{MRI Protocol}

ACQUISITION-Scan acquisition was performed on a 1.5T Philips Intera scanner at Columbia University Medical Center and transferred electronically to the University of California at Davis for morphometric analysis in the Imaging of Dementia and Aging Laboratory. For measures of white matter hyperintensities (WMH) volume, fluid-attenuated inversion recovery (FLAIR)-weighted images (repetition time $[T R]=11,000 \mathrm{~ms}$, echo time $[\mathrm{TE}]=144.0 \mathrm{~ms}$, inversion time $=2,800 \mathrm{~ms}$, field of view $[\mathrm{FOV}]=25 \mathrm{~cm}$, number of excitations $[\mathrm{nex}]=2$, matrix size $=256 \times 192$, with slice thickness $=3 \mathrm{~mm}$ ) were acquired in the axial orientation. T1-weighted images acquired in the axial plane and resectioned coronally were used to quantify hippocampus and entorhinal cortex volumes (TR $=20 \mathrm{~ms}$, $\mathrm{TE}=2.1 \mathrm{~ms}, \mathrm{FOV}=240 \mathrm{~cm}$, matrix size $=256 \times 160$, with slice thickness $=1.3 \mathrm{~mm}$ ).

MRI INFARCTS-The presence or absence of brain infarction on MRI was determined using all available images, including T1-weighted images, FLAIR-weighted images, and proton density-weighted and T2-weighted double-echo images. Only lesions $3 \mathrm{~mm}$ or larger qualified for consideration as brain infarcts. Signal void, best seen on the T2-weighted 
images, was interpreted to indicate a vessel. Other necessary imaging characteristics included cerebrospinal fluid (CSF) density on the T1-weighted image and, if the stroke was in the basal ganglia area, distinct separation from the circle of Willis vessels and perivascular spaces. Scans were further analyzed to determine the number of infarcts, their location (ie, right or left hemisphere, cortical or subcortical, and specific region), and their size. Infarcts of $1 \mathrm{~cm}$ or less were defined as small, and infarcts of more than $1 \mathrm{~cm}$ were defined as large. Two raters (blinded to the dietary assessments) determined the presence of cerebral infarction on MRI. Previously published $\kappa$ values for agreement among raters has been generally good, ranging from 0.73 to $0.90 .^{43}$

MRI WMH_-Total WMH volumes were derived on FLAIR-weighted images following a 2step process, as described. ${ }^{44,45}$ First, an operator manually traced the dura mater within the cranial vault, including the middle cranial fossa but not the posterior fossa and cerebellum. Intracranial volume was defined as the number of voxels contained within the manual tracings, multiplied by voxel dimensions and slice thickness. These manual tracings also defined the border between brain and nonbrain elements and permitted for the removal of the latter. Nonuniformities in image intensity were removed ${ }^{46}$ and 2 Gaussian probability functions, representing brain matter and CSF, were fitted to the skull-stripped image. ${ }^{44,46}$ Once brain matter was isolated, a single Gaussian distribution was fitted to the image data and a segmentation threshold for WMH was set a priori at 3.5 standard deviations (SDs) in pixel intensity above the mean of the fitted distribution of brain matter. Erosion of 2 exterior image pixels was applied to the brain matter image before modeling to remove partial volume effects and ventricular ependyma on WMH determination. WMH volume was calculated as the sum of voxels greater to or equal to $3.5 \mathrm{SD}$ above the mean intensity value of the image and multiplied by voxel dimensions and slice thickness. WMH volumes were logarithmically transformed and adjusted by intracranial volume.

\section{Predictors}

DIET-Dietary data regarding average food consumption over the past year were obtained using a 61-item version of Willett's semiquantitative food frequency questionnaire (SFFQ) (Channing Laboratory, Cambridge, MA) ${ }^{47}$ Trained interviewers administered the SFFQ in English or Spanish. We have previously reported validity (using 2-7-day food records) and reliability (using 2-3-month frequency assessments) of various components of the SSFQ in WHICAP. ${ }^{35-37}$ Dietary information was collected at baseline, which was on average 5.8 (SD 3.2) years before the MRI assessments. Although duration (years) between dietary and MRI evaluations was related neither to stroke $(p=0.77)$ nor to MeDi score $(p=0.27)$, it was included as a covariate in the analyses.

Similar to our previous work, ${ }^{2}$ we followed the method previously described by Trichopoulou and colleagues ${ }^{18}$ for the construction of the MeDi score. More specifically, we first regressed caloric intake (kcal) and calculated the derived residuals of daily gram intake (as recommended by Willet and Stampfer ${ }^{48}$ ) for each of the following 7 categories, which define the components of the MeDi as previously defined by Trichopoulou and colleagues ${ }^{18}$. dairy, meat, fruits, vegetables, legumes, cereals, and fish. A value of 0 or 1 was assigned to each of the 7 above groups, using sex-specific medians as cutoffs. For beneficial components (fruits, vegetables, legumes, cereals, and fish), persons whose consumption was below the median were assigned a value of 0 , and persons whose consumption was at or above the median was assigned a value of 1 . For components presumed to be detrimental (meat and dairy products), persons whose consumption was below the median were assigned a value of 1 , and persons whose consumption was at or above the median was assigned a value of 0 . For fat intake (eighth food category) we used the ratio of daily consumption (in grams) of monounsaturated fatty acid (MUFA) to saturated fatty acid (SFA) ${ }^{18}$ (again using 
sex-specific median cutoffs for assignment values of 0 for low and 1 for high). For alcohol intake (ninth food category), subjects were assigned a score of 0 for either no (0g/day) or more than moderate ( $\geq 30 \mathrm{~g} /$ day) consumption, and a value of 1 for mild-moderate alcohol consumption $(>0$ to $<30 \mathrm{~g} /$ day $)$. This is agreement with reports ${ }^{18}$ that consider moderate amount of alcohol consumption as another characteristic component of the MeDi. We classified alcohol consumption dichotomously also because of the skewed distribution of alcohol in our population (68\% reporting no alcohol intake, $31 \%$ reporting less than $30 \mathrm{gm} /$ day [mild to moderate intake], and $1 \%$ reporting $\geq 30 \mathrm{~g} / \mathrm{day}$ [heavy intake]). The MeDi score was generated for each participant by adding the scores in the food categories (theoretically ranging from 0 to 9 ) with higher score indicating higher adherence to the MeDi.

VASCULAR DISEASE HISTORY-History of diabetes, hypertension, and heart disease was ascertained by self-report from an interview with participants and/or their informants. ${ }^{49}$ History of heart disease included arrhythmias (eg, atrial fibrillation), coronary artery disease, and congestive heart failure. Self-report data on diabetes, hypertension, and heart disease have been shown to be reliable (test-retest reliability $\kappa=0.85$ ) and to have high sensitivity and specificity (over 90\% for either) using medical records as the gold standard in this study's cohort. ${ }^{50}$

Clinical stroke was defined according to the World Health Organization (WHO) criteria, ${ }^{51}$ based on self-report, supplemented by a neurological examination. History of clinical stroke was ascertained from an interview with participants and/or their informants. ${ }^{26}$ Positive response(s) to any 1 of the following 8 questions was considered as suggestive of a history of stroke: (1) have you ever had, (2) did a doctor tell you that you had, (3) did you have within the past year, a stroke, ministroke, cerebrovascular accident, or TIA; (4) have you ever had a sudden paralysis or numbness on 1 side of the body, (5) suddenly lost the use of speech or have slurred speech, (6) had sudden loss of consciousness with severe headache, nausea, and vomiting; (7) did the stroke or symptoms last more than 24 hours; and (8) have the stroke symptoms continued without ever going away. Persons who answered yes to any of the 8 questions were referred to see a board certified neurologist. In addition, $80 \%$ of selfreported strokes were confirmed by review of medical records.

Fasting plasma total cholesterol (TC) and triglyceride (TG) levels were determined at initial assessment using standard enzymatic techniques. High-density lipoprotein (HDL) cholesterol levels were determined after precipitation of apolipoprotein (APOE) Bcontaining lipoproteins with phosphotungstic acid. ${ }^{52}$ Low-density lipoprotein (LDL) cholesterol was recalculated using a published formula. ${ }^{53}$

COVARIATES-Age (years), education (years), caloric intake (kcal), and body mass index (weight in kilograms divided by height in square meters $\left.\left[\mathrm{kg} / \mathrm{m}^{2}\right]\right)^{54}$ were used as continuous variables. Ethnic group was based on self-report using the format of the 1990 U.S. Census. ${ }^{55}$ Participants were then assigned to 1 of 4 groups: African American (Black non-Hispanic), Hispanic, White (non-Hispanic), or Other. Ethnicity was used as a dummy variable with White (non-Hispanic) as the reference. APOE genotype was used dichotomously: absence of $\varepsilon 4$ allele vs presence of either 1 or $2 \varepsilon 4$ alleles. A trigger question inquired whether the individual ever smoked at least 1 cigarette per day for 1 year or longer. If the answer to the trigger question was no, the individual was classified as a nonsmoker and no further questions were asked. Participants who answered the question affirmatively were classified as current smokers if they were still smoking or past smokers if they had quit smoking. We finally considered a physical activity assessment. ${ }^{56}$ Briefly, self-reported time spent on a series of vigorous, moderate, and light activities was weighted by the corresponding metabolic equivalents and summed into an overall physical activity score, which was then 
categorized into tertiles: no physical activity (used as reference), some physical activity, and much physical activity (for details see Scarmeas and colleagues ${ }^{3}$ ).

\section{Statistical Analyses}

Characteristics of participants by stroke and by MeDi tertiles were compared using $t$ test or analysis of variance (ANOVA) for continuous variables and $\chi^{2}$ test for categorical variables.

BASIC MODEL-We calculated logistic regression models with MRI infarcts as the dichotomous outcome. The main predictor was MeDi score as a continuous variable initially and in tertiles form subsequently (used for trend test calculation).

ADJUSTED MODEL-In subsequent models we simultaneously adjusted for the following variables: age, sex, ethnicity, education, APOE genotype, body mass index, and duration between diet evaluation and MRI. Although caloric intake- adjusted residuals were used in the MeDi score calculation, we also included caloric intake as a covariate in the models (as recommended by Willet and Stampfer ${ }^{48}$ ).

FULLY ADJUSTED MODEL WITH VASCULAR COMORBIDITIES-To examine whether the association between MRI infarcts and MeDi was over and above clinically assessed cardiovascular comorbidity, we simultaneously included vascular risk factors (smoking, diabetes, hypertension, and heart disease) in the above adjusted logistic regression model. In a subsequent model we also added as covariates plasma TC, HDL, TG, and LDL.

SUPPLEMENTARY ANALYSES (ALL IN ADJUSTED MODELS)—We examined possible confounding effects of physical activity. We calculated sex-stratified and agestratified (using the median age as cutoff) models. In order to examine whether possible associations between MeDi and MRI infarcts was due to associations of particular food categories, we included all 9 individual components used to calculate the MeDi score as predictors. We conducted additional analyses examining separately the association of MeDi with small or large MRI infarcts, with infarct localization (right vs left hemisphere) and with number of infarcts (due to non-normal distribution we used nonparametric methods). We investigated the association of MeDi with infarcts at different locations in the brain. We examined the association of MeDi with MRI infarcts, excluding subjects with clinical strokes. Although the absence of dementia was an inclusion criterion for the WHICAP imaging study, 46 met diagnostic criteria for dementia at the clinical evaluation closest to neuroimaging. We repeated the analyses excluding these subjects with dementia. We investigated the association of MeDi with MRI infarcts excluding both subjects with clinical stroke and dementia. We finally examined the association between MeDi and WMH.

\section{Results}

\section{Clinical-Demographic-Dietary Characteristics}

MRI infarcts were detected in $31 \%$. Compared with subjects without, those with MRI infarcts were more likely to be males and to have history of hypertension or heart disease (Table 1). Subjects with MRI infarcts had higher WMH volumes. Also, they had lower MeDi scores. The groups did not differ in other demographic, anthropometric, genetic, dietary, or clinical variables.

In accordance to our previous reports, ${ }^{2}$ Hispanics adhered more and African Americans less to the MeDi (Table 2). Higher adherence to the MeDi was associated with lower rates of heart disease but not with other demographic, anthropometric, genetic, or clinical variables. 


\section{MeDi and Infarcts}

Higher adherence to the MeDi was associated with significantly lower odds of MRI infarcts (Table 3). The results were similar in adjusted and unadjusted models (models 1 and 2). Each additional unit of the MeDi score was associated with 9\% to $11 \%$ lower odds of MRI infarcts. Compared to subjects in the lowest MeDi tertile (low adherence to the MeDi), subjects in the middle MeDi score tertile had $18 \%$ to $22 \%$ lower odds of MRI infarcts, while those at the highest tertile (high adherence to the MeDi) had 36\% to 38\% lower MRI infarct odds, with a significant a trend for a dose-response effect.

Inclusion of clinical vascular comorbidity variables and physical activity did not attenuate the association (see Table 3; model 3). Additional inclusion of plasma lipid levels resulted in associations below the type I error significance level probably due to some power loss since the magnitude of the coefficients was not appreciably different.

Regarding other covariates, female sex (odds ratio [OR], 0.53; 95\% confidence interval [CI], $0.35-0.79$ ), and absence of hypertension (OR, 0.63 ; 95\% CI, 0.42-0.94) were associated with lower odds for MRI infarcts.

\section{Supplementary Analyses}

Physical activity was unrelated to both MRI infarcts and MeDi adherence (see Tables 1 and 2). Considering physical activity in adjusted models resulted in some loss of power (total $n=$ 578, physical activity not being available for all subjects) but did not appreciably change the associations: MeDi continuous OR, 0.89 (95\% CI, 0.80-0.99), $p=0.04$; middle MeDi tertile 0.75 (0.49-1.13), high MeDi tertile 0.63 (0.39-1.02), $p$ for trend 0.05 .

In sex-stratified analyses, for males only, the associations between MeDi adherence (continuous score) and MRI infarcts was 0.95 (0.80-1.14), while for females only it was $0.88(0.78-1.00)$, suggesting a slightly stronger association in women.

In age-stratified analyses, for younger subjects the association between $\mathrm{MeDi}$ adherence (continuous score) and MRI infarcts was 0.85 (0.73-0.99), while for older subjects it was 0.93 (0.81-1.08), suggesting a stronger association in younger participants.

Models that included the 9 individual dietary components of the MeDi as predictors did not reveal any statistically significant association between any individual component and MRI infarcts: dairy $0.82(0.58-1.15)$; meats $0.99(0.68-1.44)$; fruits $(0.79(0.55-1.13)$; vegetables 0.80 (0.56-1.14); legumes 1.19 (0.82-1.75); cereals 0.79 (0.55-1.14); fish $0.92(0.65-1.31)$; MUFA/SFA 0.90 (0.64-1.27); alcohol 1.25 (0.86-1.80).

There were 157 subjects who had only small MRI infarcts, 34 who had only large, and 31 who had both small and large MRI infarcts. In adjusted models, the associations between MeDi adherence (continuous score) and MRI infarcts of different size (compared to absent MRI infarcts) were as follows: only small MRI infarcts OR (95\% CI), 0.91 (0.81-1.02); only large MRI infarcts 0.83 (0.66-1.04); both small and large MRI infarcts 0.98 (0.771.24).

Infarcts were detected in the left hemisphere exclusively (no right-sided infarcts) for 62, in the right hemisphere exclusively (no left-sided infarcts) for 103, and in both right and left hemispheres for 38 subjects. In adjusted models, the associations between MeDi adherence (continuous score) were as follows: MRI infarcts exclusively on the left (vs no infarcts at all), 0.99 (0.84-1.17); MRI infarcts exclusively on the right (vs no infarcts at all), 0.91 (0.80-1.04). 
Location of infarcts were as follows (\% out of 707): basal ganglia $6 \%$, caudate $4 \%$, internal capsule $1 \%$, external capsule $3 \%$, extreme capsule $3 \%$, thalamus $3 \%$, frontal cortex $4 \%$, frontal white matter $7 \%$, parietal cortex $2 \%$, parietal white matter $4 \%$, occipital cortex $1 \%$, occipital white matter $1 \%$, temporal cortex $0.3 \%$ ( 2 subjects), temporal white matter $1 \%$, brain stem $1 \%$, and cerebellum 5\%. In adjusted models, the associations between MeDi adherence (continuous score) and presence of infarcts in each of these locations (vs no infarcts at all) were as follows: basal ganglia $0.80(0.65-0.99)$; caudate $0.99(0.76-1.25)$; internal capsule 0.63 (0.37-1.07); external capsule 0.81 (0.61-1.08); extreme capsule 0.96 (0.71-1.30); thalamus $1.11(0.83-1.50)$; frontal cortex $0.89(0.70-1.14)$; frontal white matter 0.93 (0.77-1.12); parietal cortex 0.91 (0.66-1.26); parietal white matter 0.97 (0.75-1.25); occipital cortex $0.64(0.30-1.37)$; occipital white matter $0.75(0.42-1.33)$; temporal cortex not calculable CI, $p=0.98$; temporal white matter 3.8 (0.44-30.7); brain stem 0.61 ( $0.37-$ $1.01)$; and cerebellum $0.95(0.75-1.20)$. Overall, the associations seemed stronger for brain stem and basal ganglia, internal and external capsule, occipital cortex, and white matter infarcts but data are too sparse to draw inferences.

The number of detected MRI infarcts per subject were as follows: 141 subjects had 1 infarct, 47 subjects had 2 infarcts, 16 subjects had 3 infarcts, 9 subjects had 4 infarcts, 3 subjects had 5 infarcts, 1 subject had 6 infarcts, 1 subject had 7 infarcts, 1 subject had 8 infarcts, 1 subject had 9 infarcts, 1 subject had 11 infarcts, and 1 subject had 12 infarcts. There was a borderline nonsignificant association between MeDi adherence and infarct number: higher adherence and lower infarct number; Spearman's rho $-0.73(p=0.05)$.

Excluding subjects with dementia ( $\mathrm{n}=46$, leaving 661 subjects for the analyses) did not change the associations: MeDi continuous $0.88(0.80-0.98), p=0.02$; middle MeDi tertile 0.79 (0.53-1.18), high MeDi tertile 0.59 (0.37-0.94), $p$ for trend 0.03 .

Excluding subjects with clinical stroke ( $\mathrm{n}=86$, leaving 621 subjects for the analyses) resulted in associations below the statistical significance threshold but the magnitude of the associations was not appreciably different: MeDi continuous $0.91(0.81-1.02), p=0.09$; middle MeDi tertile 0.86 (0.56-1.32), high MeDi tertile 0.62 (0.38-1.04), $p$ for trend 0.07.

When excluding subjects with both dementia and clinical stroke $(n=123$, leaving 584 subjects for the analyses) the associations between MeDi and MRI infarcts did not substantially change: MeDi continuous $0.90(0.80-1.00), p=0.07$; middle MeDi tertile 0.83 (0.53-1.28), high MeDi tertile 0.60 (0.36-1.01), $p$ for trend 0.05 .

WMH_In unadjusted models WMH were not related to MeDi (continuous MeDi score $\beta=$ $0.008 ; p=0.70$; for MeDi tertiles see Table 2). Adjusting for clinical and demographic variables (age, sex, ethnicity, education, APOE genotype, caloric intake, body mass index, and duration between diet evaluation and MRI) did not change the associations: continuous MeDi score $\beta=-0.002 ; p=0.94$; compared to low MeDi tertile (reference), middle MeDi tertile $\beta=-0.04 ; p=0.63$, high MeDi tertile $\beta=0.03 ; p=0.74 ; p$ for trend 0.78 .

Additionally adjusting for vascular variables (smoking, diabetes, hypertension, and heart disease) did not change the associations: continuous MeDi score $\beta=-0.0004 ; p=0.99$; compared to low MeDi tertile (reference), middle MeDi tertile $\beta=-0.05 ; p=0.57$, high MeDi tertile $\beta \beta=0.04 ; p=0.69 ; p$ for trend 0.73 . Results were similar when plasma lipids were additionally considered.

\section{Discussion}

We found that higher adherence to a MeDi was associated with reduced cerebrovascular disease burden. Notably, the association between MeDi and infarcts was over and above 
vascular comorbidities, including hypertension, diabetes, and heart disease. When plasma lipids were considered, the magnitude of the coefficients remained largely unchanged, suggesting that the attenuation of statistical significance was mostly due to loss of power. We found no association between MeDi and WMH.

The prevalence of MRI infarcts in our study was $31 \%$. In a recent systematic review of silent brain infarcts, ${ }^{57}$ prevalence ranged from $8 \%$ to $28 \%$ in 8 general population studies with average ages ranging from 59 to 75 years old. The biggest prevalence was noted in the Cardiovascular Health Study (mean age 75 years, mean prevalence $28 \%$ ). ${ }^{58}$ Given the known association between age and brain infarcts, our population's prevalence of MRI infarcts being slightly higher is most likely due to inclusion of older participants (average age of our population being 80 years). The inclusion of significant proportion of minorities (Hispanics and African Americans) with high burden of comorbidities could be an additional explanation of the higher prevalence of MRI infarcts in our population.

We detected no association for smoking, dyslipidemia, diabetes, or heart disease. This is not surprising because although smoking, dyslipidemia, and diabetes have been welldocumented risk factors for clinical strokes, ${ }^{27-30}$ their association with infarcts detected on imaging (which are often subclinical) has been inconsistent. ${ }^{57}$ In the present study, among cardiovascular risk factors only hypertension was associated with brain infarcts in adjusted models: subjects who did not suffer from hypertension had $40 \%$ lower odds for having brain infarcts. The association between MeDi and brain infarcts was of comparable magnitude: subjects in the highest tertile of MeDi adherence had reduction in odds for brain infarcts of about $40 \%$.

Despite the potentially protective effects for stroke of many elements of the MeDi (eg, fruits, vegetables, whole grains, fish, olive oil, low saturated fatty acids, moderate alcohol), ${ }^{27-33}$ the association between MeDi and stroke has received extremely limited attention in the previous literature. To our knowledge, there has been only 1 publication reporting a lower risk of clinical stroke in women with higher MeDi adherence. ${ }^{34}$ Our study confirms an association between MeDi and cerebrovascular disease but at the same time is the first to demonstrate an association between MeDi and subclinical infarcts captured with brain imaging.

The association between MeDi and neurodegenerative diseases has somehow received more attention. We reported that higher adherence to a MeDi is associated with lower risk for $\mathrm{AD}^{1-3}$ (same U.S. population as current study) and with lower rates of cognitive decline ${ }^{59}$ (in a French population). One of the possible biological mechanisms for the MeDi-AD association may be a vascular one: (1) higher adherence to a MeDi has been associated with lower risk for cardiovascular comorbidity, $5,6,13,14,18-20$ whereas (2) there is accumulating evidence for contribution of cardiovascular risk factors in AD risk and pathogenesis. ${ }^{22-24}$ When we examined this hypothesis in a previous report, we found that clinically assessed strokes did not seem to mediate the MeDi-AD association. ${ }^{1}$ At the same time, sensitivity and specificity of self-reported stroke (using MRI as the criterion) has been found quite low, ${ }^{26}$ possibly diminishing our ability to detect a vascular mediation in the MeDi-AD relation. ${ }^{1}$ MRI has the ability to detect and quantify cerebrovascular damage with superior sensitivity. Using MRI-assessed subclinical infarcts we now demonstrate an association between MeDi and cerebrovascular disease, suggesting that vascular mechanisms maybe in the biological pathway between MeDi and $\mathrm{AD}$. The presence of subclinical brain infarcts increases the risk for future dementia (including AD) ${ }^{60}$ and mild cognitive impairment. ${ }^{61}$ In future follow-up some of these nondemented elderly of the current study will develop dementia, which will enable us to examine explicitly whether cerebral infarction mediates the noted association between increased MeDi and decreased $\mathrm{AD}$ risk. 
With the exception of a single study that failed to detect any association between alcohol and MRI infarcts, ${ }^{62}$ to our knowledge, there is no previous literature for any other elements of our diet (either food groups or nutrients). In our own data, when individual components used to derive the MeDi pattern were examined, none was related to odds of brain infarcts. This observation mirrors our previous findings regarding AD. ${ }^{2}$ Such results strengthen even further the hypothesis that composite dietary patterns can capture dimensions of nutrition that may be missed by individual components and that an overall dietary pattern is likely to have a greater effect on health than a single nutrient.

Study limitations relating to the construction of the MeDi score (ie, use of an a priori dietary pattern score, equal weighing of underlying food categories, underestimating total food and caloric intake, etc.) have been discussed previously. ${ }^{1,2}$ The food frequency questionnaire was a brief one, while a more detailed longer instrument may have resulted in lower measurement inaccuracies/error. Dietary information was collected at baseline which was on average 5.8 (3.2 SD) years before the MRI assessments. We previously demonstrated stability of adherence to a MeDi over the course of about 8 (and up to 16) years, ${ }^{2,3}$ A subset of 1,015 individuals in WHICAP who did not develop dementia during follow-up had had multiple dietary assessments: 2 dietary assessments available for 831 participants, 3 for 137 , 4 for 43 , and 5 for 4 , with a mean time interval between dietary assessments of 6.1 years (3.1 SD; range, 1.4-16.4). The MeDi score was stable (estimated annual change of the MeDi score $\beta=-0.01, p=0.44)$. The above suggest stability over approximately a decade and that older subjects do not often change their dietary habits. Nevertheless, based on our data alone we cannot be certain about stability to MeDi adherence at middle or young age.

Additionally, the models were adjusted for duration between diet and MRI assessments but given the close proximity of the MRI and diet assessments and the observational nature of the study it would be premature to make claims about biological causality.

Infarct ratings were visually based but published $\kappa$ values for agreement among raters has been generally good, ranging from 0.73 to $0.90,{ }^{43}$ and rates were blinded to MeDi scores. This is a cohort of subjects with largely absent clinical strokes and hence the majority of infarcts were small ones. Supplementary analyses suggested a potentially stronger association between $\mathrm{MeDi}$ and large infarcts but the numbers of large infarcts are quite small to generate high confidence in this finding.

Individuals not included in the present analyses because of either missing MRI or dietary information did not differ in many characteristics but were less likely of African-American or White ethnicity, of slightly older age, and of female sex. In accordance with our previous report Hispanics adhered more and African Americans less to the $\mathrm{MeDi},{ }^{2}$ while ethnicity was not related to infarcts. Age was related neither to MeDi, nor to infarcts but stratified analyses suggested a stronger association for younger subjects. Women adhered to a MeDi equally to men but fewer of them had infarcts, while in stratified analyses the association between MeDi and infarcts was stronger for women. Overall, although we adjusted for all the above factors in our analyses and the associations between MeDi and infarcts remained unchanged, we cannot completely exclude biases due to missing data. It is also possible that diet is related to socioeconomic status or to other habits or characteristics related to better health and hence a lower risk for infarcts. In our data MeDi was not related to education or smoking but was related to ethnicity. We addressed this by adjusting for these factors but we cannot completely exclude residual confounding as an explanation for our findings. This issue can be definitively addressed only by randomized clinical trials.

Confidence in our findings is strengthened by the following factors. The population of older adults is rapidly becoming more ethnically diverse ${ }^{63}$ and the importance of including ethnic minorities in aging studies is well established. ${ }^{64}$ Even large community-based samples that 
incorporated MRI have been generally limited to Caucasians, ${ }^{43}$ with rare exception..$^{65,66} \mathrm{We}$ have previously shown that cerebrovascular disease is higher in African Americans and Hispanics than in Caucasians in our cohort. ${ }^{39}$ These observations strongly suggest that it is important to take ethnic differences in cerebrovascular disease into account. Therefore, a unique aspect of the cohort is its community-based character and its multiethnic composition with inclusion of Caucasians, Caribbean Hispanics, and African American participants, which increases the external validity of the finding. Assessment instruments (including dietary, clinical, imaging, etc.) that have been previously validated and widely used in epidemiological studies were applied. We used an a priori developed dietary pattern. ${ }^{18,67,68}$ Measures for multiple potential confounders were carefully recorded and adjusted for in the analyses. Using a variety of sensitivity analyses, the associations between $\mathrm{MeDi}$ and infarcts were essentially unchanged.

Previous studies have demonstrated that a MeDi is associated with reduced cardiovascular comorbidities. ${ }^{5-21}$ These results add to the literature by demonstrating an association of MeDi with cerebrovascular disease, specifically MRI infarcts. Our findings should be replicated and further explored.

\section{Acknowledgments}

This research was supported by grants from the NIA [AG028506 (PI: NS), PO1-AG07232 (PI: RM), AG029949 (PI: AB)]; the Taub Institute for Research on Alzheimer's Disease and the Aging Brain.

\section{References}

1. Scarmeas N, Stern Y, Mayeux R, Luchsinger JA. Mediterranean diet, Alzheimer disease, and vascular mediation. Arch Neurol. 2006; 63:1709-1717. [PubMed: 17030648]

2. Scarmeas N, Stern Y, Tang MX, et al. Mediterranean diet and risk for Alzheimer's disease. Ann Neurol. 2006; 59:912-921. [PubMed: 16622828]

3. Scarmeas N, Luchsinger JA, Schupf N, et al. Physical activity, diet, and risk of Alzheimer disease. JAMA. 2009; 302:627-637. [PubMed: 19671904]

4. Scarmeas N, Stern Y, Mayeux R, et al. Mediterranean diet and mild cognitive impairment. Arch Neurol. 2009; 66:216-225. [PubMed: 19204158]

5. Esposito K, Marfella R, Ciotola M, et al. Effect of a Mediterranean-style diet on endothelial dysfunction and markers of vascular inflammation in the metabolic syndrome: a randomized trial. JAMA. 2004; 292:1440-1446. [PubMed: 15383514]

6. Schroder H, Marrugat J, Vila J, et al. Adherence to the traditional Mediterranean diet is inversely associated with body mass index and obesity in a Spanish population. J Nutr. 2004; 134:3355-3361. [PubMed: 15570037]

7. Mendez MA, Popkin BM, Jakszyn P, et al. Adherence to a Mediterranean diet is associated with reduced 3-year incidence of obesity. J Nutr. 2006; 136:2934-2938. [PubMed: 17056825]

8. Sanchez-Villegas A, Bes-Rastrollo M, Martinez-Gonzalez MA, Serra-Majem L. Adherence to a Mediterranean dietary pattern and weight gain in a follow-up study: the SUN cohort. Int J Obes (Lond). 2006; 30:350-358. [PubMed: 16231028]

9. Buckland G, Bach A, Serra-Majem L. Obesity and the Mediterranean diet: a systematic review of observational and intervention studies. Obes Rev. 2008; 9:582-593. [PubMed: 18547378]

10. Romaguera D, Norat T, Mouw T, et al. Adherence to the Mediterranean diet is associated with lower abdominal adiposity in European men and women. J Nutr. 2009; 139:1728-1737. [PubMed: 19571036]

11. Salas-Salvado J, Fernandez-Ballart J, Ros E, et al. Effect of a Mediterranean diet supplemented with nuts on metabolic syndrome status: one-year results of the PREDIMED randomized trial. Arch Intern Med. 2008; 168:2449-2458. [PubMed: 19064829] 
12. Tortosa A, Bes-Rastrollo M, Sanchez-Villegas A, et al. Mediterranean diet inversely associated with the incidence of metabolic syndrome: the SUN prospective cohort. Diabetes Care. 2007; 30:2957-2959. [PubMed: 17712023]

13. Chrysohoou C, Panagiotakos DB, Pitsavos C, et al. Adherence to the Mediterranean diet attenuates inflammation and coagulation process in healthy adults: the ATTICA Study. J Am Coll Cardiol. 2004; 44:152-158. [PubMed: 15234425]

14. Psaltopoulou T, Naska A, Orfanos P, et al. Olive oil, the Mediterranean diet, and arterial blood pressure: the Greek European Prospective Investigation into Cancer and Nutrition (EPIC) study. Am J Clin Nutr. 2004; 80:1012-1018. [PubMed: 15447913]

15. Esposito K, Maiorino MI, Ciotola M, et al. Effects of a Mediterranean-style diet on the need for antihyperglycemic drug therapy in patients with newly diagnosed type 2 diabetes: a randomized trial. Ann Intern Med. 2009; 151:306-314. [PubMed: 19721018]

16. Martinez-Gonzalez MA, de la Fuente-Arrillaga C, Nunez-Cordoba JM, et al. Adherence to Mediterranean diet and risk of developing diabetes: prospective cohort study. BMJ. 2008; 336:1348-1351. [PubMed: 18511765]

17. Mozaffarian D, Marfisi R, Levantesi G, et al. Incidence of new-onset diabetes and impaired fasting glucose in patients with recent myocardial infarction and the effect of clinical and lifestyle risk factors. Lancet. 2007; 370:667-675. [PubMed: 17720018]

18. Trichopoulou A, Costacou T, Bamia C, Trichopoulos D. Adherence to a Mediterranean diet and survival in a Greek population. N Engl J Med. 2003; 348:2599-2608. [PubMed: 12826634]

19. de Lorgeril M, Salen P, Martin JL, et al. Mediterranean diet, traditional risk factors, and the rate of cardiovascular complications after myocardial infarction: final report of the Lyon Diet Heart Study. Circulation. 1999; 99:779-785. [PubMed: 9989963]

20. Knoops KT, de Groot LC, Kromhout D, et al. Mediterranean diet, lifestyle factors, and 10-year mortality in elderly European men and women: the HALE project. JAMA. 2004; 292:1433-1439. [PubMed: 15383513]

21. Buckland G, Gonzalez CA, Agudo A, et al. Adherence to the Mediterranean diet and risk of coronary heart disease in the Spanish EPIC Cohort Study. Am J Epidemiol. 2009; 170:1518-1529. [PubMed: 19903723]

22. Breteler MM. Vascular risk factors for Alzheimer's disease: an epidemiologic perspective. Neurobiol Aging. 2000; 21:153-160. [PubMed: 10867200]

23. Luchsinger J, Mayeux R. Cardiovascular risk factors and Alzheimer's disease. Curr Atheroscler Rep. 2004; 6:261-266. [PubMed: 15191699]

24. Cummings JL. Alzheimer's Disease. N Engl J Med. 2004; 351:56-67. [PubMed: 15229308]

25. Harris MI, Flegal KM, Cowie CC, et al. Prevalence of diabetes, impaired fasting glucose, and impaired glucose tolerance in U.S. adults. The Third National Health and Nutrition Examination Survey, 1988-1994. Diabetes Care. 1998; 21:518-524. [PubMed: 9571335]

26. Reitz C, Schupf N, Luchsinger JA, et al. Validity of self-reported stroke in elderly African Americans, Caribbean Hispanics, and Whites. Arch Neurol. 2009; 66:834-840. [PubMed: 19433651]

27. European Stroke Organisation (ESO) Executive Committee; ESO Writing Committee. Guidelines for management of ischaemic stroke and transient ischaemic attack 2008. Cerebrovasc Dis. 2008; 25:457-507. [PubMed: 18477843]

28. Goldstein LB, Adams R, Alberts MJ, et al. Primary prevention of ischemic stroke: a guideline from the American Heart Association/American Stroke Association Stroke Council: cosponsored by the Atherosclerotic Peripheral Vascular Disease Interdisciplinary Working Group; Cardiovascular Nursing Council; Clinical Cardiology Council; Nutrition, Physical Activity, and Metabolism Council; and the Quality of Care and Outcomes Research Interdisciplinary Working Group: the American Academy of Neurology affirms the value of this guideline. Stroke. 2006; 37:1583-1633. [PubMed: 16675728]

29. Galimanis A, Mono ML, Arnold M, et al. Lifestyle and stroke risk: a review. Curr Opin Neurol. 2009; 22:60-68. [PubMed: 19155763]

30. Rundek T, Sacco RL. Risk factor management to prevent first stroke. Neurol Clin. 2008; 26:10071045. ix. [PubMed: 19026901] 
31. Ding EL, Mozaffarian D. Optimal dietary habits for the prevention of stroke. Semin Neurol. 2006; 26:11-23. [PubMed: 16479440]

32. He FJ, Nowson CA, MacGregor GA. Fruit and vegetable consumption and stroke: meta-analysis of cohort studies. Lancet. 2006; 367:320-326. [PubMed: 16443039]

33. Ricci S, Celani MG, Righetti E, et al. Fatty acid dietary intake and the risk of ischaemic stroke: a multicentre case-control study. UFA Study Group. J Neurol. 1997; 244:360-364. [PubMed: 9249620]

34. Fung TT, Rexrode KM, Mantzoros CS, et al. Mediterranean diet and incidence of and mortality from coronary heart disease and stroke in women. Circulation. 2009; 119:1093-1100. [PubMed: 19221219]

35. Luchsinger JA, Tang MX, Shea S, Mayeux R. Caloric intake and the risk of Alzheimer disease. Arch Neurol. 2002; 59:1258-1263. [PubMed: 12164721]

36. Luchsinger JA, Tang MX, Shea S, Mayeux R. Antioxidant vitamin intake and risk of Alzheimer disease. Arch Neurol. 2003; 60:203-208. [PubMed: 12580704]

37. Luchsinger JA, Tang MX, Siddiqui M, et al. Alcohol intake and risk of dementia. J Am Geriatr Soc. 2004; 52:540-546. [PubMed: 15066068]

38. Scarmeas N, Levy G, Tang MX, et al. Influence of leisure activity on the incidence of Alzheimer's disease. Neurology. 2001; 57:2236-2242. [PubMed: 11756603]

39. Tang MX, Cross P, Andrews H, et al. Incidence of Alzheimer's disease in African-Americans, Caribbean Hispanics and Caucasians in northern Manhattan. Neurology. 2001; 56:49-56. [PubMed: 11148235]

40. Brickman AM, Schupf N, Manly JJ, et al. Brain morphology in older African Americans, Caribbean Hispanics, and whites from northern Manhattan. Arch Neurol. 2008; 65:1053-1061. [PubMed: 18695055]

41. Stern Y, Andrews H, Pittman J, et al. Diagnosis of dementia in a heterogeneous population. Development of a neuropsychological paradigm-based diagnosis of dementia and quantified correction for the effects of education. Arch Neurol. 1992; 49:453-460. [PubMed: 1580806]

42. American Psychiatric Association. Diagnostic and statistical manual of mental disorders. 3rd ed.. Washington, DC: American Psychiatric Press; 1987.

43. DeCarli C, Massaro J, Harvey D, et al. Measures of brain morphology and infarction in the Framingham heart study: establishing what is normal. Neurobiol Aging. 2005; 26:491-510. [PubMed: 15653178]

44. DeCarli C, Maisog J, Murphy DG, et al. Method for quantification of brain, ventricular, and subarachnoid CSF volumes from MR images. J Comput Assist Tomogr. 1992; 16:274-284. [PubMed: 1545026]

45. DeCarli C, Murphy DG, Tranh M, et al. The effect of white matter hyperintensity volume on brain structure, cognitive performance, and cerebral metabolism of glucose in 51 healthy adults. Neurology. 1995; 45:2077-2084. [PubMed: 7501162]

46. DeCarli C, Murphy DG, Teichberg D, et al. Local histogram correction of MRI spatially dependent image pixel intensity nonuniformity. J Magn Reson Imaging. 1996; 6:519-528. [PubMed: 8724419]

47. Willett WC, Sampson L, Stampfer MJ, et al. Reproducibility and validity of a semiquantitative food frequency questionnaire. Am J Epidemiol. 1985; 122:51-65. [PubMed: 4014201]

48. Willett, W.; Stampfer, M. Implications of total energy intake for epidemiological analyses. In: Willett, W., editor. Nutritional epidemiology. New York: Oxford University Press; 1998. p. 273-301.

49. Luchsinger JA, Reitz C, Honig LS, et al. Aggregation of vascular risk factors and risk of incident Alzheimer disease. Neurology. 2005; 65:545-551. [PubMed: 16116114]

50. Luchsinger JA, Tang MX, Shea S, Mayeux R. Hyperinsulinemia and risk of Alzheimer disease. Neurology. 2004; 63:1187-1192. [PubMed: 15477536]

51. Hatano S. [Plans for prevention of stroke formulated by WHO and practice in Japan]. Nippon Rinsho. 1976; 34:131-136. [PubMed: 943009] 
52. Lopes-Virella MF, Stone P, Ellis S, Colwell JA. Cholesterol determination in high-density lipoproteins separated by three different methods. Clin Chem. 1977; 23:882-884. [PubMed: 192488]

53. Friedewald WT, Levy RI, Fredrickson DS. Estimation of the concentration of low-density lipoprotein cholesterol in plasma, without use of the preparative ultracentrifuge. Clin Chem. 1972; 18:499-502. [PubMed: 4337382]

54. Kuczmarski R, Carroll M, Flegal K, Troiano R. Varying body mass index cutoff points to describe overweight prevalence among U.S. adults: NHANES III (1988 to 1994). Obes Res. 1997; 5:542548. [PubMed: 9449138]

55. Census of Population and Housing. Summary Tape File1, Technical Documentation. Washington, DC: U.S. Bureau of the Census; 1991.

56. Godin G, Shephard RJ. A simple method to assess exercise behavior in the community. Can J Appl Sport Sci. 1985; 10:141-146. [PubMed: 4053261]

57. Vermeer SE, Longstreth WT Jr, Koudstaal PJ. Silent brain infarcts: a systematic review. Lancet Neurol. 2007; 6:611-619. [PubMed: 17582361]

58. Price TR, Manolio TA, Kronmal RA, et al. Silent brain infarction on magnetic resonance imaging and neurological abnormalities in community-dwelling older adults. CHS Collaborative Research Group. Stroke. 1997; 28:1158-1164. [PubMed: 9183343]

59. Feart C, Samieri C, Rondeau V, et al. Adherence to a Mediterranean diet, cognitive decline, and risk of dementia. JAMA. 2009; 302:638-648. [PubMed: 19671905]

60. Vermeer SE, Prins ND, den Heijer T, et al. Silent brain infarcts and the risk of dementia and cognitive decline. N Engl J Med. 2003; 348:1215-1222. [PubMed: 12660385]

61. Lopez OL, Jagust WJ, Dulberg C, et al. Risk factors for mild cognitive impairment in the Cardiovascular Health Study Cognition Study: part 2. Arch Neurol. 2003; 60:1394-1399. [PubMed: 14568809]

62. Ding J, Eigenbrodt ML, Mosley TH Jr, et al. Alcohol intake and cerebral abnormalities on magnetic resonance imaging in a community-based population of middle-aged adults: the Atherosclerosis Risk in Communities (ARIC) study. Stroke. 2004; 35:16-21. [PubMed: 14657449]

63. U.S. Census Bureau. U.S. Interim Projections by Age, Sex, Race, and Hispanic Origin. Washington, DC: U.S. Census Bureau; 2004.

64. Brown DR, Alexander M. Recruiting and retaining people of color in health research studies: introduction. J Aging Health. 2004; 16:S5-S8.

65. Lopez OL, Kuller LH, Fitzpatrick A, et al. Evaluation of dementia in the cardiovascular health cognition study. Neuroepidemiology. 2003; 22:1-12. [PubMed: 12566948]

66. Mosley TH Jr, Knopman DS, Catellier DJ, et al. Cerebral MRI findings and cognitive functioning: the Atherosclerosis Risk in Communities study. Neurology. 2005; 64:2056-2062. [PubMed: 15985571]

67. Trichopoulos D, Lagiou P. Mediterranean diet and cardiovascular epidemiology. Eur J Epidemiol. 2004; 19:7-8. [PubMed: 15012017]

68. Jacques PF, Tucker KL. Are dietary patterns useful for understanding the role of diet in chronic disease? Am J Clin Nutr. 2001; 73:1-2. [PubMed: 11124739] 


\section{TABLE 1}

Demographic, Clinical, and Dietary Characteristics

\begin{tabular}{|c|c|c|c|c|}
\hline Characteristics & $\begin{array}{l}\text { MRI infarcts } \\
\text { absent }(n=485)\end{array}$ & $\begin{array}{l}\text { MRI infarcts } \\
\text { present(n = 222) }\end{array}$ & $\operatorname{All}(n=707)$ & $p^{a}$ \\
\hline Age, yr, mean (SD) & $80.4(5.5)$ & $80.1(5.9)$ & $80.3(5.7)$ & 0.61 \\
\hline Sex: M, n (\%) & $147(30)$ & $92(41)$ & $239(34)$ & 0.004 \\
\hline Education, yr, mean (SD) & $10.4(4.9)$ & $11.0(4.6)$ & $10.6(4.8)$ & 0.13 \\
\hline Ethnicity, n (\%) & & & & 0.15 \\
\hline White & $126(26)$ & $66(30)$ & $192(27)$ & \\
\hline African American & $165(34)$ & $85(38)$ & $250(35)$ & \\
\hline Hispanic & $182(38)$ & $69(31)$ & $251(36)$ & \\
\hline Other & $12(3)$ & $2(1)$ & $14(2)$ & \\
\hline Presence of $\varepsilon 4$ allele (\%) & $112(24)$ & $60(28)$ & $172(26)$ & 0.28 \\
\hline Energy (kcal), mean (SD) & $1,451.6(499.0)$ & $1,473.3(581.3)$ & $1,458.4(525.9)$ & 0.61 \\
\hline Body mass index, mean (SD) & $27.7(5.8)$ & $27.0(5.1)$ & $27.5(5.6)$ & 0.09 \\
\hline Smoking, n (\%) & & & & 0.68 \\
\hline Past & $165(35)$ & $83(38)$ & $248(36)$ & \\
\hline Current & $66(14)$ & $30(14)$ & $96(14)$ & \\
\hline Physical activity, n (\%) & & & & 0.95 \\
\hline None & $109(25)$ & $47(24)$ & $156(25)$ & \\
\hline Some & $143(33)$ & $66(34)$ & $209(33)$ & \\
\hline Much & $178(41)$ & $82(42)$ & $260(42)$ & \\
\hline Diabetes, n (\%) & $103(21)$ & $57(26)$ & $160(23)$ & 0.19 \\
\hline Hypertension, n (\%) & $313(65)$ & $160(72)$ & $473(67)$ & 0.05 \\
\hline Heart disease, $\mathrm{n}(\%)$ & $95(20)$ & $59(27)$ & $154(22)$ & 0.04 \\
\hline $\mathrm{TC}, \mathrm{mg} / \mathrm{dl}$, mean (SD) & $202.0(36.9)$ & $196.6(39.8)$ & $200.3(37.9)$ & 0.10 \\
\hline $\mathrm{HDL}, \mathrm{mg} / \mathrm{dl}$, mean $(\mathrm{SD})$ & $49.9(14.8)$ & $47.7(15.2)$ & $49.2(15.0)$ & 0.09 \\
\hline $\mathrm{TG}, \mathrm{mg} / \mathrm{dl}$, mean (SD) & $145.6(71.1) 150.3(82.6)$ & $150.3(82.6)$ & $147.0(74.8)$ & 0.47 \\
\hline LDL, mg/dl, mean (SD) & $123.0(32.3)$ & $118.9(35.2)$ & $121.8(33.3)$ & 0.15 \\
\hline MRI WMH, mean (SD) & $-4.9(0.82)$ & $-4.4(0.90)$ & $-4.7(0.87)$ & $<0.001$ \\
\hline MeDi score, mean (SD) & $4.5(1.7)$ & $4.2(1.7)$ & $4.4(1.7)$ & 0.05 \\
\hline MeDi tertiles, n (\%) & & & & 0.11 \\
\hline Low & $141(29)$ & $80(36)$ & $221(31)$ & \\
\hline Middle & $206(43)$ & $92(41)$ & $298(42)$ & \\
\hline High & $138(29)$ & $50(21)$ & $188(27)$ & \\
\hline
\end{tabular}

${ }^{a}$ Bold indicates significant $(<0.05)$ values of $p$.

$\mathrm{HDL}=$ high density lipoprotein cholesterol; $\mathrm{LDL}=$ low density lipoprotein cholesterol; $\mathrm{MeDi}=$ Mediterranean diet; $\mathrm{MRI}=$ magnetic resonance imaging; $\mathrm{SD}=$ standard deviation; $\mathrm{TC}$ = total cholesterol; TG = triglycerides; $\mathrm{WMH}=$ white matter hyper-intensities (logarithmically transformed ratio to total intracranial volume). 
TABLE 2

Demographic and Clinical Characteristics by MeDi Score Tertiles

\begin{tabular}{|c|c|c|c|c|}
\hline Characteristics & $\begin{array}{l}\text { Low Tertile } \\
\text { (MeDi score 0-3) }\end{array}$ & $\begin{array}{l}\text { Middle Tertile } \\
\text { (MeDi score 4-5) }\end{array}$ & $\begin{array}{l}\text { High Tertile } \\
\text { (MeDi score 6-9) }\end{array}$ & $p^{a}$ \\
\hline Age, yr, mean (SD) & $79.6(5.4)$ & $80.4(5.8)$ & $80.9(5.7)$ & 0.07 \\
\hline Sex: M, n (\%) & $75(34)$ & $105(35)$ & $59(31)$ & 0.68 \\
\hline Education, yr, mean (SD) & $10.8(4.8)$ & $10.8(4.6)$ & $10.0(5.1)$ & 0.12 \\
\hline Ethnicity, n (\%) & & & & 0.003 \\
\hline White & $64(29)$ & $75(25)$ & $53(28)$ & \\
\hline African American & $92(42)$ & $111(37)$ & $47(25)$ & \\
\hline Hispanic & $63(29)$ & $107(36)$ & $81(43)$ & \\
\hline Other & $2(1)$ & $5(2)$ & $7(4)$ & \\
\hline Presence of $\varepsilon 4$ allele (\%) & $56(28)$ & $66(23)$ & $50(28)$ & 0.38 \\
\hline Energy (kcal), mean (SD) & $1,478.7(549.9)$ & $1,448.6(513.2)$ & $1,450.1(519.1)$ & 0.79 \\
\hline Body mass index (SD) & $27.8(6.1)$ & $27.6(5.5)$ & $26.9(5.1)$ & 0.25 \\
\hline Smoking, n (\%) & & & & 0.15 \\
\hline Past & $84(40)$ & $102(35)$ & $62(34)$ & \\
\hline Current & $35(17)$ & $42(15)$ & $19(10)$ & \\
\hline Physical activity, n (\%) & & & & 0.46 \\
\hline None & $51(27)$ & $71(27)$ & $34(20)$ & \\
\hline Some & $64(34)$ & $85(32)$ & $60(35)$ & \\
\hline Much & $75(39)$ & $107(41)$ & $78(45)$ & \\
\hline Diabetes, n (\%) & $49(22)$ & $68(23)$ & $43(23)$ & 0.98 \\
\hline Hypertension, n (\%) & $148(67)$ & $197(66)$ & $128(68)$ & 0.90 \\
\hline Heart disease, $\mathrm{n}(\%)$ & $62(28)$ & $59(20)$ & $33(18)$ & 0.02 \\
\hline $\mathrm{TC}, \mathrm{mg} / \mathrm{dl}$, mean (SD) & $199.3(35.3)$ & $199.3(37.4)$ & $203.2(41.5)$ & 0.53 \\
\hline $\mathrm{HDL}, \mathrm{mg} / \mathrm{dl}$, mean $(\mathrm{SD})$ & $49.0(46.2)$ & $48.6(14.2)$ & $50.3(14.7)$ & 0.51 \\
\hline $\mathrm{TG}, \mathrm{mg} / \mathrm{dl}$, mean (SD) & $146.2(81.3)$ & $150.8(71.3)$ & $142.2(72.2)$ & 0.51 \\
\hline $\mathrm{LDL}, \mathrm{mg} / \mathrm{dl}$, mean $(\mathrm{SD})$ & $121.0(31.8)$ & $120.5(33.3)$ & $124.5(34.9)$ & 0.46 \\
\hline MRI infarcts, n (\%) & $80(36)$ & $92(31)$ & $50(27)$ & 0.11 \\
\hline MRI WMH, mean (SD) & $-4.8(0.91)$ & $-4.8(0.86)$ & $-4.7(0.86)$ & 0.70 \\
\hline
\end{tabular}

${ }^{a}$ Bold indicates significant $(<0.05)$ values of $p$.

$\mathrm{HDL}=$ high density lipoprotein cholesterol $; \mathrm{LDL}=$ low density lipoprotein cholesterol $; \mathrm{M}=$ male $; \mathrm{MeDi}=$ Mediterranean diet $; \mathrm{MRI}=$ magnetic resonance imaging; $\mathrm{SD}=$ standard deviation; $\mathrm{TC}=$ total cholesterol; $\mathrm{TG}=$ triglycerides; $\mathrm{WMH}=$ white matter hyperintensities (logarithmically transformed ratio to total intracranial volume). 


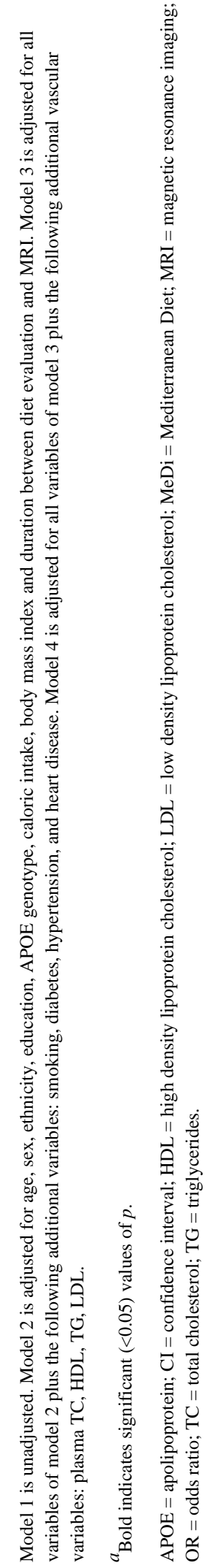

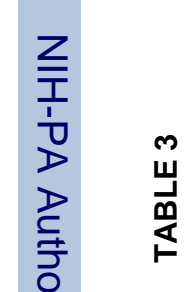

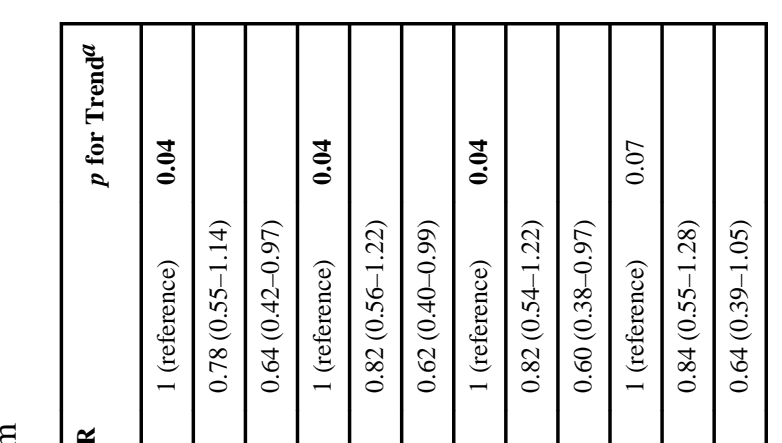

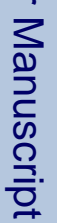

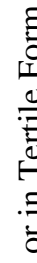

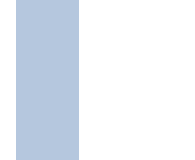

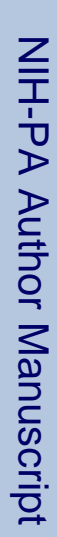

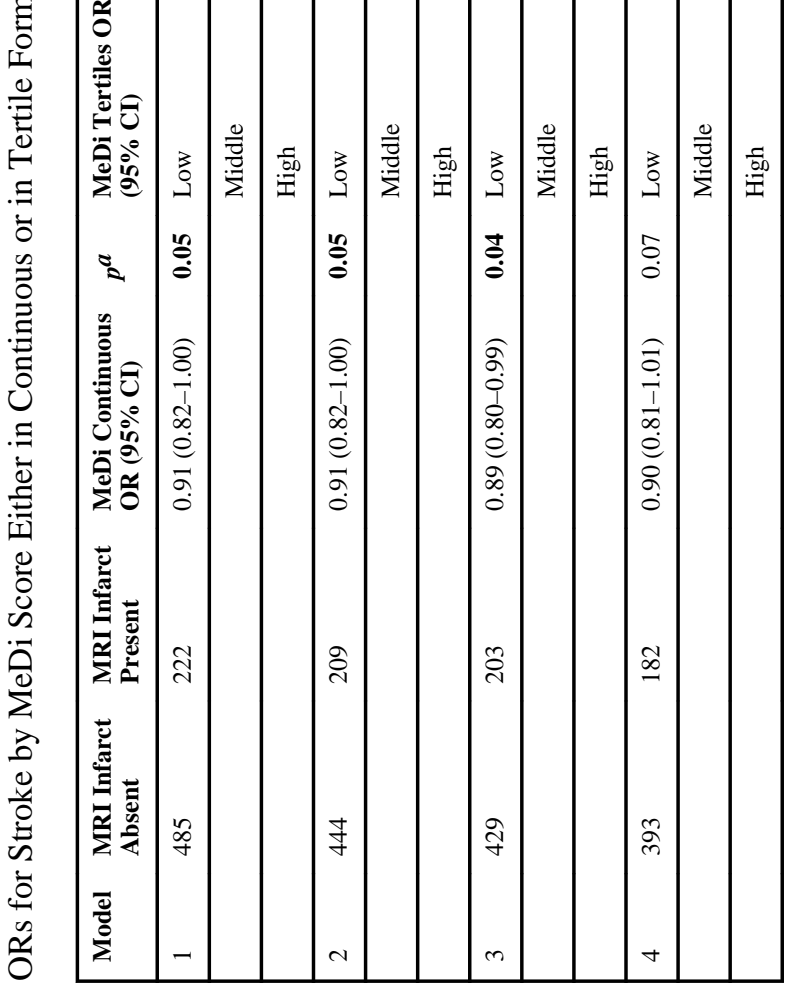

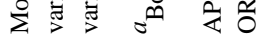

